SVU- International Journal of Veterinary Sciences, 4(1): 54-65, 2021.

Print ISSN: 2535-1826

\title{
Histopathological changes of kidney tissue during aging
}

\section{Mohamed H. Kotob ${ }^{1 *}$, Ahmed M. Hussein ${ }^{2}$, Mahmoud Abd-Elkareem ${ }^{3}$}

${ }^{1}$ Department of Pathology and Clinical Pathology, Faculty of Veterinary Medicine, Assiut University, Assiut, Egypt. ${ }^{2}$ Zoology Department, Faculty of Science, Al-Azhar University, Assiut, Egypt. ${ }^{3}$ Department of Anatomy, Histology and Embryology, Faculty of Veterinary Medicine, Assiut University, Assiut, Egypt.

\section{Abstract}

Kidney aging is a normal physiological process associates with various molecular, morphologic and functional changes in the kidney tissues. This work was designed to study microscopically the structural changes in the kidney tissue of aged rats compared to young rats. Male Sprague-Dawley rats were used 10 young rats (4 months) and 10 aged rats (24 months). Rats were transcardially perfused with $4 \%$ paraformaldehyde. The kidneys were post fixed for 24 hours in $4 \%$ PFA then proceeded for normal histopathology and light microscopic examination. Kidney tissue of aged rats showed serious morphological changes such as segmental glomerulosclerosis, pericapsular fibrosis, tubulointerstitial fibrosis, perivascular fibrosis, inflammatory cell infiltration, tubular dilatation, intra-tubular cast formation and tubular atrophy. These changes were compared to the normal histological appearance of glomeruli, tubules, interstitium and blood vessels of young rat kidneys. In addition, the kidney tissue of the aged rats showed compensatory glomerular hypertrophy, tubular hyperplasia and endothelial proliferation. Renal aging involves several degenerative changes in kidney structure and these alterations interfere with the physiologic functions and end with chronic renal failure.

Keywords: Aging, Kidney, Glomerulosclerosis, Tubulointerstitial fibrosis, Rats.

DOI: 10.21608/svu.2021.55868.1092 Received: December30, 2020 Accepted: February 3, 2021 Published: March 24, 2021 Corresponding Author: Mohamed H. Kotob: E-mail: Mohamedkotob@vet.aun.edu.eg.

Citation: Kotob et al., 2021. Histopathological changes of kidney tissue during aging. SVU-IJVS 2021, 4(1): 54-65.

Copyright: (c) Kotob et al. This is an open access article distributed under the terms of the creative common attribution license, which permits unrestricted use, distribution and reproduction in any medium provided the original author and source are created.

Competing interest: The authors have declared that no competing interest exists. 


\section{INTRODUCTION}

Aging is a natural progressive and inevitable physiological process associated with several molecular, structural and functional changes in different body organs (Muthuswamy et al., 2006; Glass et al., 2013). Oxidative stress and lipid peroxidation resulted from the accumulation of cellular oxidative free radicals are considered important pathways and mechanisms behind the aging process (Rahal et al., 2014). Recently, some studies have focused on the mitochondrial role during tissue aging that includes mitochondrial oxidative stress and mitochondrial damage (Dai et al., 2014).

The macroscopic anatomical changes during kidney aging mostly include decline in the kidney volume due to the decrease of the renal cortical volume and increase of renal medullary volume (Wang et al., 2014; Denic et al., 2016; Fang et al., 2020). Senile kidney also contains simple numerous cysts that does not cause kidney enlargement (Hommos et al., 2017). The surface of aged kidney appears more granular and rough because of the global glomerulosclerosis, interstitial fibrosis and tubular atrophy of the outer renal cortex (Silva, 2005; Zhou et al., 2008).

The microscopic histological changes of the aged kidney are thought to develop from the arteriosclerosis of renal blood vessels during aging. The arteriosclerosis is considered as an initiating factor for the nephropathy in aged kidney and consists of arterial intimal fibrosis and thickening, medial hypertrophy and vascular hyalinosis. These vascular changes cause an ischemic nephropathy and lead to focal glomerulosclerosis in the form of pericapsular fibrosis, thickening of glomerular capillary basement membrane, wrinkling glomerulus and synechiae of glomeruli with Bowman's capsule (Zhou et al., 2008; Rule et al., 2010; Denic et al., 2016). Later on, tubulointerstitial changes appear and consist of thickening of basement membranes, intratubular casts and tubular atrophy due to interstitial fibrosis (Thomas et al., 1998; Zhou et al., 2008; Denic et al., 2016; Schlanger et al., 2020). These micro-anatomical changes lead to collapsing of glomerular tuft, development of diffuse sclerotic glomeruli and finally decrease the number of functional glomeruli with compensatory hypertrophy of rest of existing nephron (Kubo et al., 2003; Martin and Sheaff, 2007; Denic et al., 2016). The degenerative apoptotic changes and decrease numbers of kidney podocytes were also recorded during aging (Zhang et al., 2012; Wiggins, 2012). Some cases develop other pathological changes like interstitial calcifications, focal cortical scars and fibromuscular dysplasia (Lorenz et al., 2010).

These structural changes in the kidney affect the renal functions causing decrease in the glomerular filtration rate, alterations in the capillary wall permeability, changes in renal tubular reabsorptions and urinary concentration and affect the hormonal production role of the kidney (Esposito and Dal Canton, 2010; Wiggins, 2012; Denic et al., 2016).

This research work was designed to study the histopathological changes occurred in the kidney tissue of aged rats compared to young rats.

\section{MATERIALS AND METHODS}

\section{Animals}

Male Sprague-Dawley rats were used in these experiment 10 young rats (average $400 \mathrm{~g}, 4$ months old), 10 old rats (average $650 \mathrm{~g}, 24$ months old).

Rats were housed and maintained in a separate room (3 rats/ cage) throughout the 
experiment. Rats were kept under standard laboratory conditions at a temperature of $21 \pm 2{ }^{\circ} \mathrm{C}$ with a humidity of $50 \pm 5 \%$ and 12 $\mathrm{h} / 12 \mathrm{~h}$ dark/light cycle (light on at 7:00 am). Rats were fed ad libitum with free access to water. The study was carried out under the guideline of the Ethics Committee, Faculty of Veterinary Medicine, Assiut university, Egypt.

\section{Animal Perfusion and histological preparation}

Rats were anesthetized with $40 \mathrm{mg} / \mathrm{kg}$ IP pentobarbital. Intracardiac perfusion was done for all rats using ice-cold phosphate buffered saline $(0.1 \mathrm{M})$ at a $\mathrm{pH}$ of 7.5 for approximately $10 \mathrm{~min}$ followed by perfusion using 4\% paraformaldehyde (PFA) in 0.1 M PBS. After that, the whole organs were collected and post-fixed for 24 $\mathrm{h}$ in the same 4\% PFA then processed to prepare paraffin blocks. Kidneys were sectioned using Leica microtome at $5 \mu \mathrm{m}$ then stained for histological study with:

\section{Histological stains}

1) Hematoxyline and Eosin stain (Fischer et al., 2008).

2) Periodic acid-Schiff stain (Aterman and Norkin, 1963).

3) Sirius Red stain (Bhutda et al., 2017).

4) Masson's trichrome stain (Flint et al., 1975).

All staining preparations were examined by an Olympus BX51 microscope and the photos were taken by an Olympus DP72 camera adapted into the microscope.

\section{Negative image analysis using CMEIAS color segmentation}

Negative image analysis was performed using CMEIAS color segmentation to assess the complex color micrographs that were obtained to give more details (AbdElkareem, 2017; Abd-Elkareem et al., 2020).

\section{RESULT}

Kidneys of young and aged rats were examined by H\&E, PAS, Sirius Red and Masson's trichrome stains. Histologically, the kidney of young rats (4 months) showed normal architecture of renal corpuscle, proximal tubules and distal tubules. The renal corpuscle was formed of normal-sized glomerulus, mesangium and Bowman's capsule. The glomerulus was a tuft of blood capillaries surrounded by double walled Bowman's capsule. It was composed of several capillary loops which lined by endothelial cells and rest on PAS positive glomerular basement membrane. Bowman's capsule was formed of parietal layer and visceral layer separated by a Bowman's space. The continuous healthy parietal layer of Bowman's capsule was formed of single layer of simple squamous epithelium rest on PAS positive capsular basement membrane. While the visceral layer was formed of podocytes. The proximal tubules were formed of intensely acidophilic large cuboidal cells with welldeveloped PAS positive brush borders and rounded centrally located nuclei. It had a narrow lumen and thin PAS positive tubular basement membrane. While the distal tubules were formed of small lightly stained cuboidal cells with wide lumen and apical rounded nuclei. Juxtaglomerular apparatus was consisted of the macula densa cells, polar cushion cells and Juxtaglomerular cells. Macula densa cells of juxtaglomerular apparatus were modified epithelial cells of the distal convoluted tubules close to the vascular pole of its glomerulus. These cells were numerous tall columnar cells with deeply stained nuclei. Polar cushion cells or extraglomerular mesangial cells were a 
group of polyhedral cells occupies a triangular area between afferent, efferent arterioles and distal tubule at the vascular pole. Juxtaglomerular cells were secretory modified smooth muscle cells of tunica media of the afferent arteriole with round nuclei (Fig. 1A, B; Fig. 2 A, B; Fig. 4 A, C, E; Fig. 5 A, C, E; Fig. 6 A, C, E).

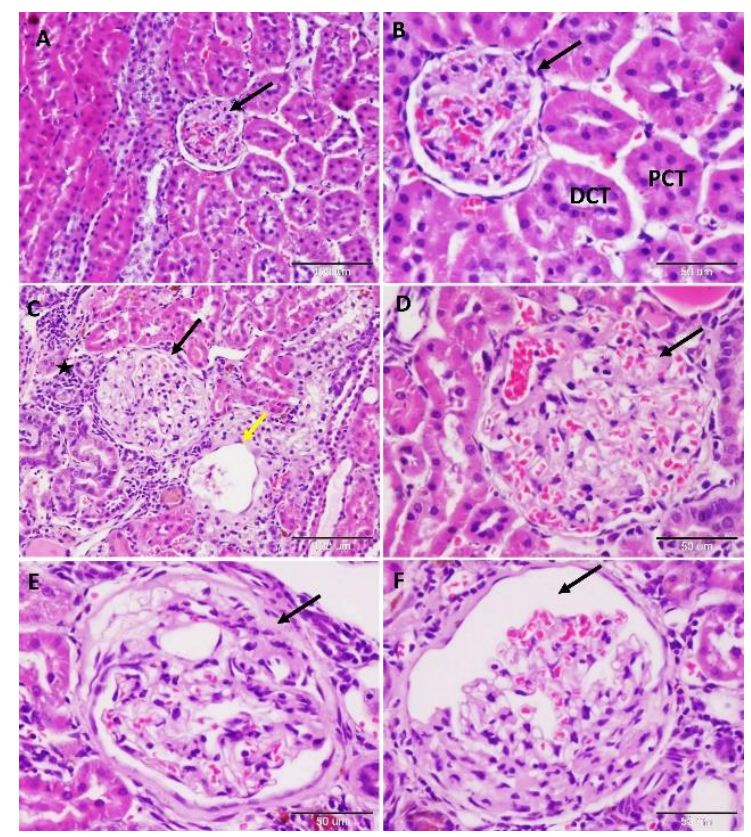

Figure 1: Glomerular changes associated with kidney aging: A, B: photomicrographs of young rat kidneys showing normal corpuscular structure (normal glomerular capillary tuft, mesangium and Bowman's capsule) (arrow) and normal proximal convoluted tubule (PCT) and Distal convoluted tubule (DCT). C, D, E, F: Photomicrographs of aged rat kidneys; C: Showing asymmetry in glomerular size and shape, one hypertrophied (black arrow) and other atrophied with cystic appearance and atrophy of glomerular tuft (yellow arrow) and inflammatory cells infiltration (star), D: Showing glomerulus with thickened basement membrane, mesangial cell proliferation and absence of Bowman's space (arrow), E: Showing segmental glomerulosclerosis characterized by periglomerular fibrosis and capillary wrinkling (Arrow), F: Showing segmental sclerotic glomerulus with destroyed capillary tuft (arrow). H \& Eosin stain. Scale bar $=100 \mu \mathrm{m}$ in A, $\mathrm{C} ; 50 \mu \mathrm{m}$ in $\mathrm{B}, \mathrm{D}, \mathrm{E}, \mathrm{F}$.

\section{Glomerular changes}

Examination of glomeruli of aged rats (24 months old) exhibited the presence of various degrees of segmental glomerulosclerosis (SGS). Aged rat kidneys showed asymmetry in glomerular size and shape, some glomeruli decreased in size with atrophied glomerular tuft and cystic appearance while the others hypertrophied and increased in size as a compensatory mechanism (Fig. 1C). The glomeruli of aged rats were characterized by mesangial cell proliferation, thickened glomerular capillary tuft and absence of Bowman's space (Fig. 1 C, D). Segmental thickening of glomerular capillary basement membranes, glomerular wrinkling, capillary collapsing and synechiae of glomeruli with Bowman's capsule are noticed (Fig. 1F). Periglomerular fibrosis of Bowman's capsule are also found (Fig. 1E).

\section{Tubulointerstitial changes}

Examination of renal tubulointerstitium of young rats (4 months) by $H \& E$ stain revealed the normal histology of tubular epithelium and interstitium (Fig. 2 A, B).

However, the kidneys of aged rats (24 months) showed a pronounced tubulointerstitial fibrosis with large areas showing tubular dilatation and intratubular cast formation (Fi. 2 C). Large amount of connective tissue and mononuclear inflammatory cells were infiltrated in the interstitium causing widening of interstitium, peritubular fibrosis and tubular atrophy (Fig. 2 D).

Tubular epithelium was flattened and showed various degrees of degeneration and apoptosis and appeared as cystic dilatation (Fig. $2 \mathrm{~F}$ ) and contained intracellular brown lipofuscin pigments with areas of hemorrhage streaks (Fig. 2E). 


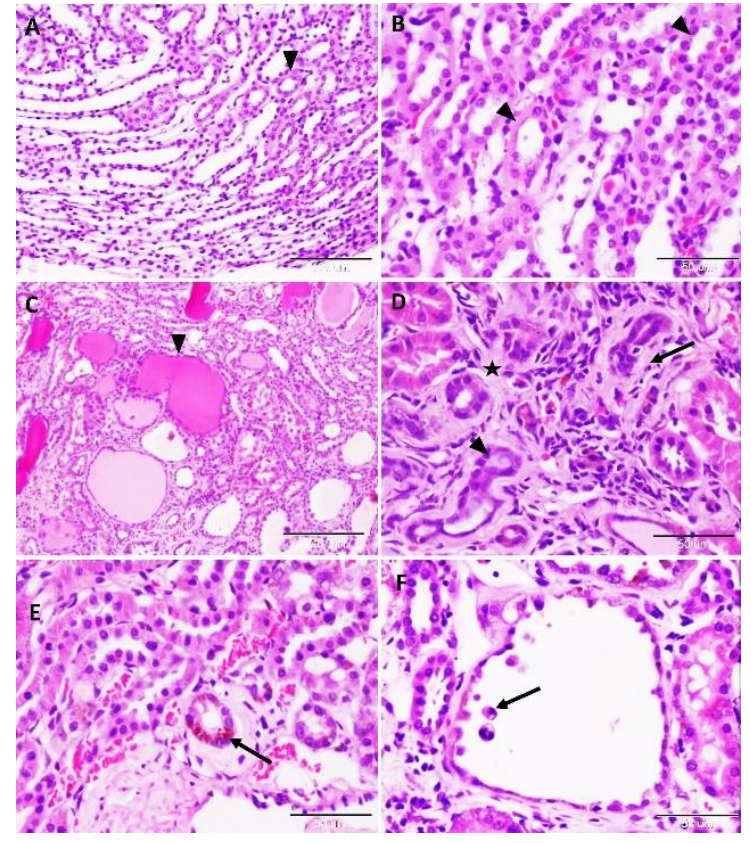

Figure 2: Tubulointerstitial changes associated with kidney aging: A, B: Photomicrographs of young rat kidneys showing normal tubules (arrowhead) and interstitium. C, D, E, F: Photomicrographs of aged rat kidneys; C: Showing tubular dilation with intratubular hyaline cast formation (arrowhead), D: Showing interstitial fibrosis (star) with excess collagen, inflammatory cell infiltration (arrow) and tubular atrophy (arrowhead), E: Showing tubular epithelium with peritubular fibrosis and contains intracellular golden brown lipofuscin granules (arrow), F: Showing tubular cyst and apoptotic bodies in tubular lumen (arrow). H \& Eosin stain. Scale bar $=100 \mu \mathrm{m}$ in A, C; $50 \mu \mathrm{m}$ in B, D, E, F.

\section{Compensatory renal changes}

The aged rat kidneys showed compensatory glomerular hypertrophy, tubular hyperplasia and vascular endothelial proliferation (Fig. $3 \mathrm{~A}, \mathrm{~B}$ ).

\section{Juxtaglomerular apparatus changes}

The aged rat kidneys showed degenerated macula densa cells, degenerated polar cushion cells (PC) and degenerated Juxtaglomerular cells (Fig. 3C).

\section{Hydropic degeneration}

The renal tubular epithelial cells showed hydropic degeneration and peripherally located nuclei (Fig. 3D).

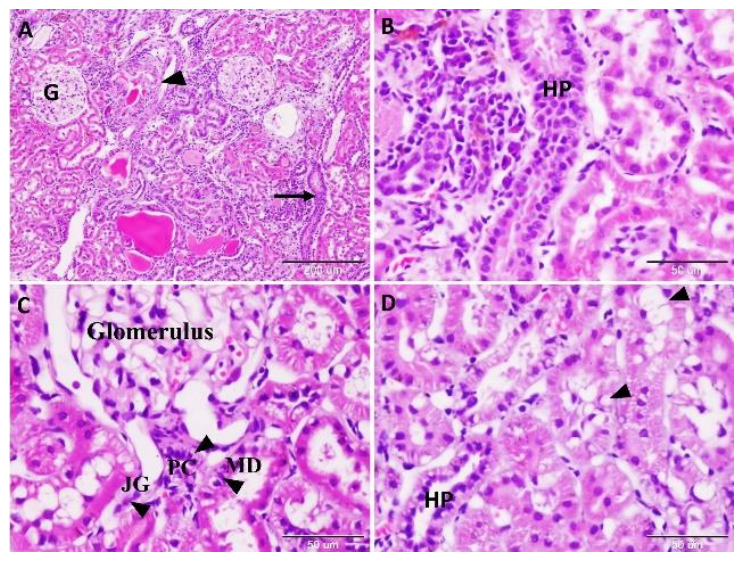

Figure 3: Nephrogenic changes associated with kidney aging: A, B, C, D: Photomicrographs of aged rat kidneys. A: showing glomerular hypertrophy $(G)$, tubular hyperplasia (arrow) and vascular intimal proliferation (arrowhead). B: Showing tubular hyperplasia (HP). C: Showing degenerated Juxtaglomerular apparatus (Arrowhead); degenerated macula densa cells (MD), degenerated polar cushion cells (PC) and degenerated Juxtaglomerular cells (JG). D: Showing tubular hyperplasia (HP) and hydropic degeneration in the renal tubular epithelial cells (arrowhead). H \& Eosin stain. Scale bar $=200 \mu \mathrm{m}$ in A; $50 \mu \mathrm{m}$ in B, C, D.

\section{Deposition of collagen and thickening of basement membranes}

PAS staining confirmed the normal histology of young rat kidneys and showed normal Bowman's capsule, glomerular capillary and tubular basement membranes with thickened tubular brush border (Fig. 4 A, C, E). However, kidneys of aged rats (24 months) revealed thickening of Bowman's capsule, glomerular capillary and tubular basement membranes with thinning of tubular brush border (Fig. 4 B, D, F).

Segmental glomerulosclerosis and tubulointerstitial fibrosis of aged rat kidneys were confirmed by the specific stains Sirius Red and Masson's trichrome 


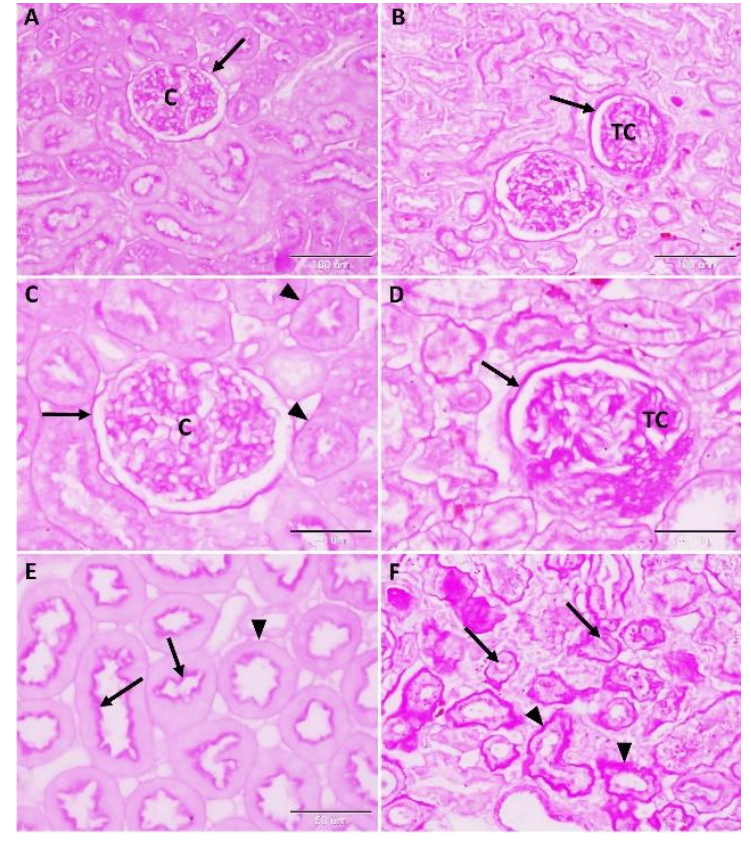

Figure 4: Histopathological changes of the aged kidney by PAS stain: A, C, E: Photomicrographs of young rat kidneys; (A, C): Showing normal appearance of glomerular capillary tuft (C) and Bowman's capsule basement membrane (arrow); (E): Showing normal renal tubules with thick brush border (arrow) and normal tubular basement membrane (arrowhead). B, D, F: Photomicrographs of aged rat kidneys; (B, D): Showing thickened glomerular capillary basement membrane (TC) and Bowman's capsule basement membrane (arrow); (F): Showing thickened basement membrane of renal tubules (arrowhead) and damaging of brush borders (arrow). Periodic acid-Schiff stain (PAS). Scale bars $=100 \mu \mathrm{m}$ in A, B; $50 \mu \mathrm{m}$ in C, D, E, F.

stains that showed mesangial matrix expansion and accumulation of collagenous fibers in the periglomerular, perivascular, peritubular and interstitium areas (Fig. 5, 6 and 7 B, D, F). Sirius Red and Masson's trichrome stains for young rat kidneys confirmed the normal glomerular structure and renal interstitium with no collagen accumulation in the interstitium or in the wall of blood vessels (Fig. 5, 6 and 7 A, C, E). We summarized the histopathological lesions appeared in the old rats compared to young rats in table 1 .

Table 1: Showing lesion score in percentages for the pathological changes in the examined kidneys of old rats compared to young rats

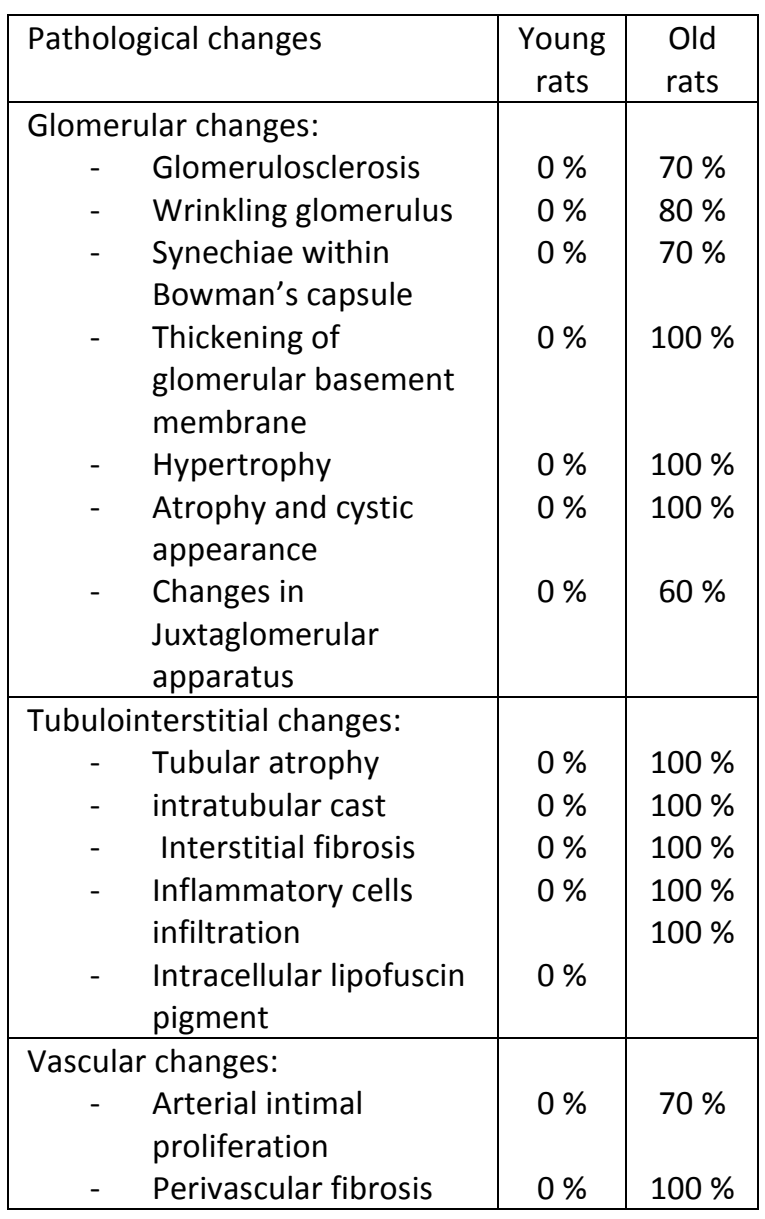

\section{DISCUSSION}

As time goes by, the senile kidney undergoes numerous structural and functional changes. In the current study, the results show a dramatic change in the glomerular structure characterized microscopically by segmental glomerulosclerosis, pericapsular fibrosis, mesangium expansion, thickening of glomerular capillary basement membrane, wrinkling glomerulus and synechiae of glomeruli with Bowman's capsule. 


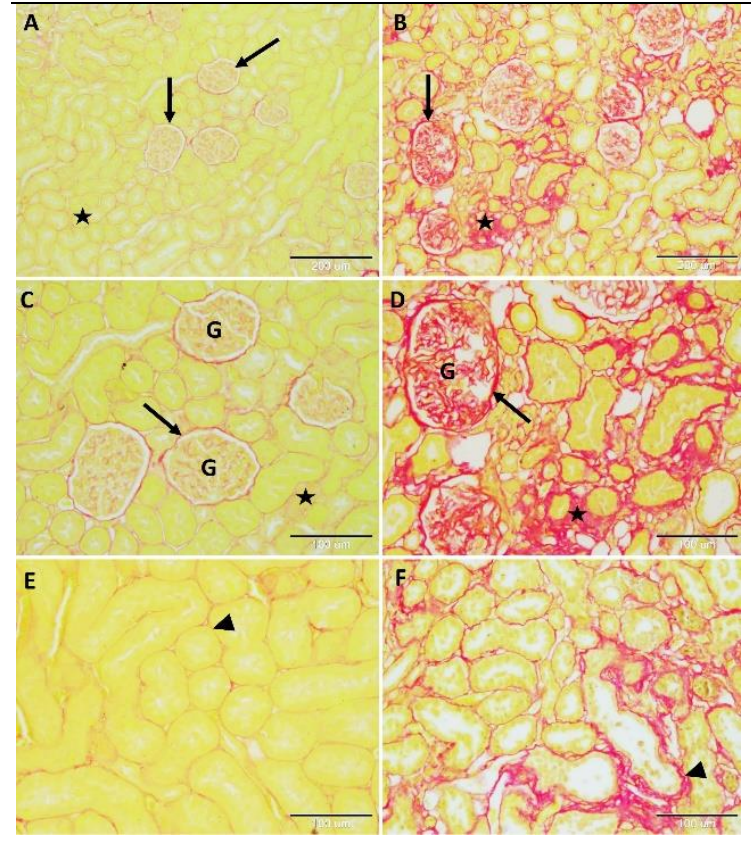

Figure 5: Histopathological changes of the aged kidney by Sirius Red stain: A, C, E: photomicrographs of young rat kidneys showing normal Bowman's capsule basement membrane (arrow) and glomerular architecture (G), normal interstitium (star) and normal tubular basement membrane (arrowhead). B, D, F: Photomicrographs of aged rat kidneys showing tubulointerstitial fibrosis (star), pericapsular fibrosis (arrow), mesangial matrix expansion $(G)$ and peritubular fibrosis (arrowhead). Sirius Red stain. Scale bar = $200 \mu \mathrm{m}$ in $\mathrm{A}, \mathrm{B} ; 100 \mu \mathrm{m}$ in $\mathrm{C}, \mathrm{D}, \mathrm{E}, \mathrm{F}$.

Glomerular size varies; sclerotic glomeruli appeared small and atrophied while the rest enlarged and hypertrophied causing asymmetry in the glomerular size. These alterations are congruent with the changes mentioned by Nishida et al., (1996); Rodrfguez-Puyol D, (1998); Zhou et al., (2008) and Schlanger et al., (2020). Finally, the sclerotic glomeruli may be completely re-absorbed, or remain atrophied to tiny small size that difficult to be detect by renal biopsy samples (Hoy et al., 2003).

The glomerular structural changes are followed by deterioration in the functional changes like decreasing of glomerular filtration rate, renal blood flow and crossing
SVU-IJVS, 4 (1): 54-65

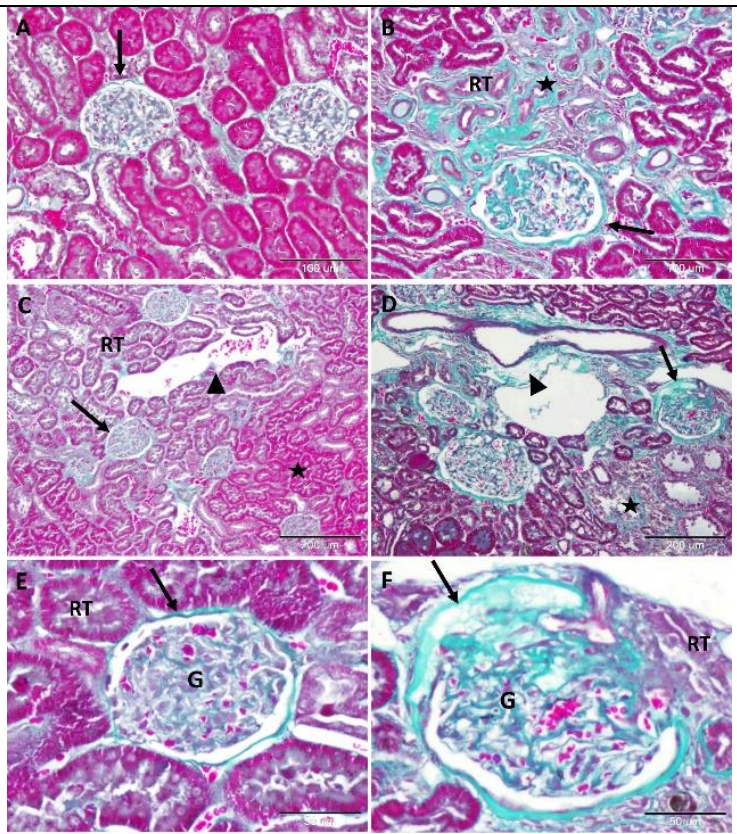

Figure 6: Histopathological changes of the aged kidney by Masson's Trichrome stain: A, C, E: Photomicrographs of young rat kidneys showing normal glomerular (G) and Bowman's capsule basement membranes (arrow), normal renal interstitium (star), renal tubules (RT) and wall of the blood vessels appears also normal (arrow head). B, D, F: Photomicrographs of aged rat kidneys showing the interstitial fibrosis (star) and atrophied renal tubules (RT), periglomerular fibrosis (segmental glomerulosclerosis) (arrow) and perivascular fibrosis (arrowhead). Masson's Trichrome stain. Scale bar $=100 \mu \mathrm{m}$ in A, B; 200 $\mu \mathrm{m}$ in $\mathrm{C}, \mathrm{D} ; 50$ in $\mathrm{E}, \mathrm{F}$.

of large proteins for the glomerular filtration barrier forming intratubular hyaline casts that demonstrated microscopically (Zhou et al., 2008).

Degeneration of cells in the juxtamedullary area results in degeneration and atrophy of the afferent and efferent arterioles and the formation of a direct channel between these two arterioles causing decreasing of cortical blood flow (Sobamowo and Prabhakar, 2017).

Tubulointerstitial changes are also present and consist of tubulointerstitial fibrosis, apoptotic and atrophying changes in convoluted tubules and macrophages / 
lymphocytes interstitial infiltration. The rest of tubules hypertrophied, dilated and contain intratubular casts in their lumen. These tubulointerstitial changes in the aged kidney were in agreement with the findings mentioned by Thomas et al. (1998) and Zhou et al., (2008).

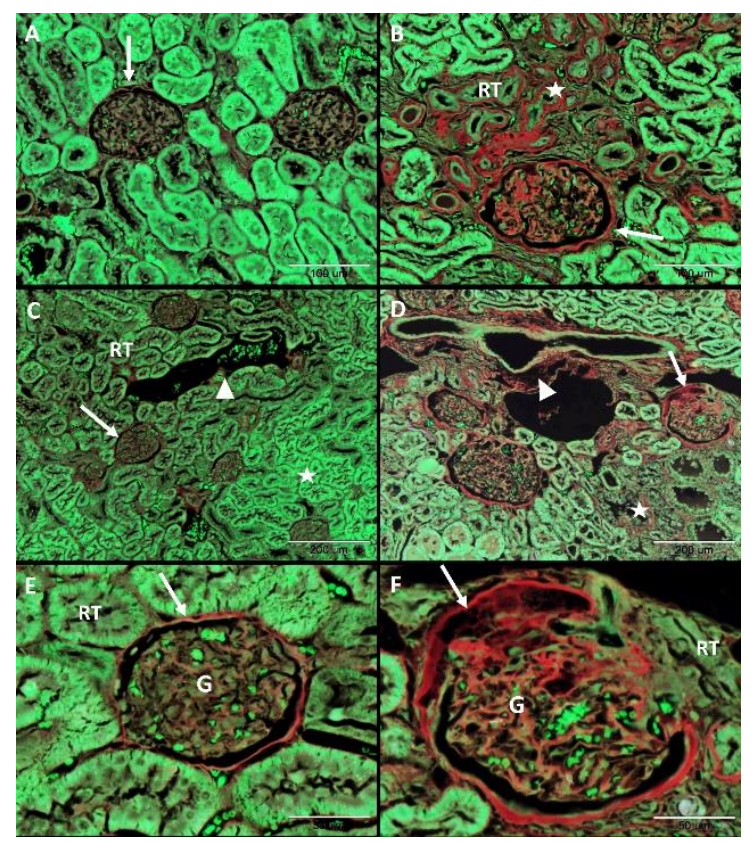

Figure 7: Negative images of the photomicrographs shown in Fig. 6.

Our results demonstrated the presence of arterial intimal fibrosis in the aged kidneys that led to renal ischemia and developed to segmental glomerulosclerosis, tubulointerstitial fibrosis and other ageassociated renal changes and this explanation based on the previous research that have similar results (Newbold et al., 1992; Thomas et al., 1998; Zhou et al., 2008).

We found also the incidence of glomerulosclerosis and tubular atrophy with advancing age is greater than the compensatory hypertrophy of non-sclerotic glomeruli and their related tubules resulting in decreasing of kidney volume (Goyal, 1982).
Nitric oxide (NO) synthase (NOS), is a catalytic enzyme its function to produce NO. NOS is mostly expressed in central and peripheral neurons and endothelial cells of blood vessels. Endothelial NOS (eNOS) maintains the vasodilatation of blood vessels has anti-atherosclerotic role and vasoprotective effects (Förstermann and Sessa, 2012). Thomas et al. (1998) found by immunohistochemistry that the aged rats (24 months) revealed a relative loss of the immunostaining of eNOS, RECA-1 and $\mathrm{OX}-2$ in peritubular capillaries in areas of tubulointerstitial injury compared to young rats (3 months). This loss of expression of eNOS is accountable for the peritubular capillary injury and ischemia in kidney tissue that initiate the aging-related tubulointerstitial fibrosis.

On the other hand, Thomas et al. (1998) also mentioned that old rat kidneys demonstrated the presence of eNOS in renal tubular epithelium and infiltrating inflammatory cells in areas of tubulointerstitial injury.

Kidneys of senile rats demonstrated presence of apoptosis process in the tubular epithelium and apoptotic bodies were noticed in the tubular lumen. These findings were previously mentioned and confirmed by Thomas et al. (1998) that found an increase in number TUNEL-positive cells in tubular and interstitial cells in the aged rats compared to young using TUNEL assay technique for apoptosis.

Periodic acid-Schiff stain showed the thickening of glomerular capillary basement membrane and, Bowman's capsule and this explained by Abrass et al. (1995) who found kidneys of aged rats are characterized by changes in the extracellular matrix (ECM) composition like increasing the immunostaining for thrombospondin, laminin chains B1 and slaminin and this responsible for the 
increasing of basement membrane thickness.

The renal tubules showed thickening of basement membranes and thinning of their brush border due to degenerative and apoptotic changes of the epithelium and these are responsible for the decline in the tubular reabsorption function of senile kidney.

Abrass et al. (1995) found that aged rats with interstitial fibrosis developed various changes in the ECM composition near to the atrophied tubules in form of increased the expression of thrombospondin, fibronectin (FN) and collagen I and III. These previous findings are congruent with and explain our current results of interstitial fibrosis and tubular atrophy.

Increasing of apoptosis in the tubular epithelium during kidney aging might be due to decrease in level of SIRT1 (a NAD ${ }^{+}$- dependent protein deacetylase). SIRT1 diminishes and inhibits the intracellular hypoxia-induced fibrosis, apoptosis via $\mathrm{p} 53$ deacetylation and inflammation. Therefore, declining level of SIRT1 during aging is one of the mechanisms behind the increasing levels of apoptosis (Kume et al., 2006).

\section{CONCLUSIONS}

Aging Kidney undergoes serious structural and functional changes with time. These structural changes are segmental glomerulosclerosis, tubulointerstitial fibrosis, inflammatory cellular infiltration, intratubular cast formation and tubular atrophy. Further studies in the near future should be directed for deep understanding the cellular mechanisms behind aging process in attempts to decrease or prevent these physiologic alterations during senility.

\section{CONFLICT OF INTEREST}

The authors declare that they have no conflict of interest

\section{REFERENCES}

Abd-Elkareem M (2017). Cell-specific immuno-localization of progesterone receptor alpha in the rabbit ovary during pregnancy and after parturition. Animal Reproduction Science, 180: 100120.

Abd-Elkareem M, Abou Khalil NS, Sayed AE-DH (2020). Cytoprotective effect of Nigella sativa seed on 4nonylphenol-induced renal damage in the African catfish (Clarias gariepinus). Chemosphere, 259: 127379.

Abrass CK, Adcox MJ, Raugi GJ (1995). Aging-associated changes in renal extracellular matrix. The American journal of pathology, 146 (3): 742.

Aterman K, Norkin S (1963). The Periodic Acid - Schiff Reaction. Nature, 197:1306-1306.

Bhutda S, Surve MV, Anil A, Kamath K, Singh N, Modi D, Banerjee A (2017). Histochemical Staining of Collagen and Identification of Its Subtypes by Picrosirius Red Dye in Mouse Reproductive Tissues. BioProtocol, 7: e2592-e2592.

Dai DF, Chiao YA, Marcinek DJ, Szeto HH, Rabinovitch PS (2014). Mitochondrial oxidative stress in aging and healthspan. Longevity \& healthspan, 3 (1): 1-22. 
Denic A, Glassock RJ, Rule AD (2016). Structural and functional changes with the aging kidney. Advances in chronic kidney disease, 23 (1): 1928.

Esposito C, Dal Canton A (2010). Functional changes in the aging kidney. Journal of nephrology, 23 Suppl 15:S41-5.

Fang Y, Gong AY, Haller ST, Dworkin LD, Liu Z, Gong R (2020). The ageing kidney: Molecular mechanisms and clinical implications. Ageing research reviews, 101151.

Fischer AH, Jacobson KA, Rose J, Zeller R (2008). Hematoxylin and Eosin Staining of Tissue and Cell Sections. Cold Spring Harbor. Protocol, 2008(5): pdb.prot4986.

Flint MH, Lyons MF, Meaney MF, Williams DE (1975). The Masson staining of collagen - an explanation of an apparent paradox. The Histochemical. Journal, 7(6): 529-546.

Förstermann U, Sessa WC (2012). Nitric oxide synthases: regulation and function. European heart journal, 33 (7): 829-837.

Glass D, Viñuela A, Davies MN, Ramasamy A, Parts L, Knowles D, Brown AA, Hedman ÅK, Small KS, Buil A, Grundberg E (2013). Gene expression changes with age in skin, adipose tissue, blood and brain. Genome biology, 14 (7):R75.
Goyal VK (1982). Changes with age in the human kidney. Experimental gerontology, 17 (5): 321-331.

Hommos MS, Glassock RJ, Rule AD (2017). Structural and functional changes in human kidneys with healthy aging. Journal of the American Society of Nephrology, 28 (10): 2838-2844.

Hoy WE, Douglas-Denton RN, Hughson MD, Cass A, Johnson K, Bertram JF (2003). A stereological study of glomerular number and volume: preliminary findings in a multiracial study of kidneys at autopsy. Kidney International, 63: S31-S37.

Kubo M, Kiyohara Y, Kato I, Tanizaki Y, Katafuchi R, Hirakata H, Okuda S, Tsuneyoshi M, Sueishi K, Fujishima M, Iida M (2003). Risk factors for renal glomerular and vascular changes in an autopsybased population survey: the Hisayama study. Kidney international, 63 (4): 1508-1515.

Kume S, Haneda M, Kanasaki K, Sugimoto T, Araki SI, Isono M, Isshiki K, Uzu T, Kashiwagi A, Koya D (2006). Silent information regulator 2 (SIRT1) attenuates oxidative stress-induced mesangial cell apoptosis via p53 deacetylation. Free Radical Biology and Medicine, 40 (12): 2175-2182.

Lorenz EC, Vrtiska TJ, Lieske JC, Dillon JJ, Stegall MD, Li X, Bergstralh EJ, Rule AD (2010). Prevalence of renal artery and kidney abnormalities by computed tomography among healthy adults. 
Clinical Journal of the American Society of Nephrology, 5 (3): 431438.

Martin JE, Sheaff MT (2007). Renal ageing. The journal of Pathology, 211 (2):198-205.

Muthuswamy AD, Vedagiri K, Ganesan M, Chinnakannu P (2006). Oxidative stress-mediated macromolecular damage and dwindle in antioxidant status in aged rat brain regions: Role of Lcarnitine and DL- $\alpha$-lipoic acid. Clinica chimica acta, 368 (1-2):8492.

Newbold KM, Sandison A, Howie AJ (1992). Comparison of size of juxtamedullary and outer cortical glomeruli in normal adult kidney. Virchows Archiv A, 420 (2): 127 129.

Nishida E, Yamanouchi J, Ogata S, Itagaki S, Doi K (1996). Age-related histochemical and ultrastructural changes in renal glomerular mesangium of APA hamsters. Experimental animals, 45 (4): 339345.

Rahal A, Kumar A, Singh V, Yadav B, Tiwari R, Chakraborty S, Dhama K (2014). Oxidative stress, prooxidants, and antioxidants: the interplay. BioMed research international, 2014: 761264.

Rodrfguez-Puyol D (1998). The aging kidney. Kidney international, 54(6): 2247-2265.

Rule AD, Amer H, Cornell LD, Taler SJ, Cosio FG, Kremers WK, Textor
SC, Stegall MD (2010). The

association between age and nephrosclerosis on renal biopsy among healthy adults. Annals of internal medicine, 152 (9): 561567.

Schlanger, L.E., Bailey, J.L. and Sands, J.M. 2020. Aging and the Kidney: Clinical and Pathophysiologic Issues. In: Chronic Renal Disease.2nd Ed. Academic Press, pp: 249-262.

Silva FG (2005). The aging kidney: a review-part I. International urology and nephrology, 37 (1): 185-205.

Sobamowo, H. and Prabhakar, S.S., 2017. The kidney in aging: physiological changes and pathological implications. In: Progress in molecular biology and translational science, Vol. 146, Academic Press, pp: 303-340.

Thomas SE, Anderson S, Gordon KL, Oyama TT, Shankland SJ, Johnson RJ (1998). Tubulointerstitial disease in aging: evidence for underlying peritubular capillary damage, a potential role for renal ischemia. Journal of the American Society of Nephrology, 9 (2): 231242.

Wang X, Vrtiska TJ, Avula RT, Walters LR, Chakkera HA, Kremers WK, Lerman LO, Rule AD (2014). Age, kidney function, and risk factors associate differently with cortical and medullary volumes of the kidney. Kidney international, 85(3): 677-685. 
Wiggins JE (2012). Aging in the

glomerulus. Journals of

Gerontology Series A: Biomedical

Sciences and Medical Sciences, 67

(12): 1358-1364.

Zhang J, Hansen KM, Pippin JW, Chang

AM, Taniguchi Y, Krofft RD,

Pickering SG, Liu ZH, Abrass CK,

Shankland SJ (2012): De novo

expression of podocyte proteins in parietal epithelial cells in experimental aging nephropathy. American Journal of PhysiologyRenal Physiology, 302 (5): F571F580.

Zhou XJ, Rakheja D, Yu X, Saxena R, Vaziri ND, Silva FG (2008). The aging kidney. Kidney international, 74 (6): 710-720. 